Filo. y Lingüí. 9(2): 83-86, 1984.

\title{
ALGUNAS CONSIDERACIONES SOBRE LA INNUMERABILIDAD DE LOS PRONUNCIAMIENTOS LINGUISTICOS
}

Manuel Arce Arenales

La escuela lingùi ística estructuralista (o "taxonómica") distingue tradicionalmente cuatro niveles del lenguaje: el nivel fonético, el fonemático, el morfológico y el sintáctico. Para enmarcar adecuadamente la discusión posterior, es útil hacer una síntesis descriptiva de la construcción de cada nivel.

\section{Nivel Fonético:}

Sea $\mathrm{S}$ el conjunto de todos los sonidos producibles por el aparato fonador humano. Incluyamos en S una "unidad de silencio", un "no-sonido", y designémoslo mediante un símbolo, al igual que a los sonidos. Sean ahora $p, q \in S$. Entonces, si no existe lenguaje humano (esto incluye, en teoría, cualquier lengua pasada, presente o futura) tal que p y q sean utilizables para diferenciar el significado de dos palabras $\mathrm{w}_{1}$ y $\mathrm{w}_{2}$ donde $\mathrm{w}_{1}=\alpha_{1} \alpha_{2} \ldots$ $\alpha_{i} \quad$ p $\alpha_{i}+1 \ldots \alpha_{n}, w_{2}=\alpha_{1} \alpha_{2} \ldots \alpha_{i}$ q $\alpha_{i}+1 \ldots$ $\alpha_{n}, \alpha_{\mathrm{j}} \in \mathrm{S} \forall \mathrm{j}=1, \ldots, \mathrm{i}, \mathrm{i}+1, \ldots \mathrm{n}$, entonces se dice que $\mathrm{p}$ y $\mathrm{q}$ son equivalentes. Sea $\mathrm{P}$ el conjunto de todas las clases de equivalencia formadas sobre $\mathrm{S}$ de la manera anterior. Entonces decimos que $\mathrm{P}$ es el conjunto de todos los "fonos", y cada elemento de $\mathrm{P}$ es un fono.

\section{Nivel Fonemático:}

Para cualquier lenguaje $L$, si $p, q \in P$ y no existen dos palabras distintas $w_{1} y w_{2}$ tales que $\mathrm{w}_{1}=\pi_{1} \ldots \pi_{\mathrm{i}} \mathrm{p} \pi_{\mathrm{i}}+1 \ldots \pi_{\mathrm{n}}, \mathrm{w}_{2}=\pi_{1} \ldots \pi_{\mathrm{i}} \mathrm{q} \pi_{\mathrm{i}}+1 \ldots$ $\pi_{n}, \pi_{j} \in P \forall j=1, \ldots, i, i+1, \ldots n$, entonces se dice que $p$ y $q$ son equivalentes para $L$ y $P_{L}$ es el conjunto de todas las clases de equivalencia así formadas sobre $P$. Los elementos de $P_{L}$ se conocen como los "fonemas" del lenguaje L. Dos fonos equivalentes se Ilaman "alófonos" del fonema al cual pertenecen.

\section{Nivel Morfológico:}

Considérese $\mathrm{L}$ y su conjunto de fonemas asociado $P_{L}$. Sea ahora $P_{L}^{*}=\left\{w / w=\beta_{1} \beta_{2} \ldots \beta_{m}\right.$, $\left.\beta_{i} \in P_{L} \nabla_{*} w=\phi, m \in N\right\}$. Consideremos entonces $w \in P_{L}^{*}$. Si $w$ es significativo $y$ no es posible reducirlo sin eliminar su significación (i.e., no es posible encontrar algún $w^{\prime} \subset w_{\text {tal que }} w^{\prime}$ sea significativo), diremos que w es un "morfo". El conjunto de todos los morfos del $\mathrm{L}$ puede designarse mediante $M_{L}^{*} \subset P_{L}^{*}$. Si ahora consideramos $a, b \in M_{L}^{*} y$ no existen dos oraciones distintas $\sigma_{1}$ y $\sigma_{2}$ tales que $\sigma_{1}=\beta_{1} \ldots \beta_{1} a_{i}+\beta_{1} \ldots \beta_{m}$, $\sigma_{2}=\beta_{1} \ldots \beta_{i} b \beta_{i}+1 \ldots \beta_{m}, \beta_{j} \in M_{L}^{*} \quad j=1, \ldots$, $\mathrm{i}, \mathrm{i}+1, \ldots, \mathrm{m}$, entonces se dice que a y $\mathrm{b}$ son equivalentes para $\mathrm{L}$, y $_{\mathrm{L}}$ es el conjunto de todas las clases de equivalencia así formadas sobre $M_{L}^{*}$. Los elementos de $M_{1}$ se conocen como los "morfemas" del lenguaje L. Dos morfos equivalentes se llaman "alomorfos" del morfema al cual pertenecen.

\section{Nivel Sintáctico:}

Sea $\Sigma_{L}^{*}$ el conjunto de todas las oraciones sobre L. Supongamos ahora que determinamos el conjunto de todos aquellos morfemas $x$ tales que $\sigma=\gamma_{1} \ldots \gamma_{\mathrm{i}} \times \gamma_{\mathrm{i}+1} \ldots \gamma_{\mathrm{n}}$ sea una oración válida $\left(\sigma \in \Sigma_{\mathrm{L}}^{*}, \gamma_{\mathrm{j}} \in \mathrm{M}_{\mathrm{L}} \forall \mathrm{j}=1, \ldots, \mathrm{i}, \mathrm{i}+1, \ldots, \mathrm{n}\right.$.) Designemos este conjunto mediante el símbolo $\mathrm{M}_{\mathrm{L}}^{X} \cdot \mathrm{Si}$ podemos encontrar otras oraciones $\sigma^{*}=\delta_{1} \ldots \delta_{\mathrm{j}} \ldots \delta_{\mathrm{m}}$ para las cuales exista algún $\mathrm{j}$ tal que $\delta_{\mathrm{j}}$ sea sustituible por cada uno de los elementos de $M_{L}^{x}, y \sigma^{*}$ siga siendo una oración válida, estableceremos $\mathrm{M}_{\mathrm{L}}^{\mathrm{X}}$ como una clase de equivalencia denominada 
"clase léxica". Una vez conocidas todas las clases léxicas de $L$, digamos $M_{L}^{1}, M_{L}^{2}, \ldots, M_{L}^{x}$, podemos establecer el conjunto $\Sigma_{L}$ de secuencias de morfemas válidas para $L$. Estas secuencias pueden denotarse mediante fórmulas como la siguiente: $M_{L}^{i}+M_{L}^{j}+M_{L}^{k}$, con $i, j, k, \in\{1,2, \ldots, x\}$.

La restricción general de los gramáticos estructuralistas (en ocasiones llamada la "condición taxonómica"- (1) ), es que para cualquier nivel dado $i$, una entidad descrita a ese nivel debe ser una clase de elementos tales que cada uno de ellos sea una entidad descrita previamente a nivel $\mathrm{i}-\mathrm{I}$.

Esto genera una restricción metodológica (conocida por algunos como la "condición operacional"- (2) ), según la cual el lingüista debe proceder como sigue:

\section{PASO 1:}

Para un lenguaje dado L, obtenga un "corpus" suficientemente rico en enunciaciones en los niveles 1 y 2 , y proceda a establecer el conjunto $P_{L}$.

\section{PASO 2:}

Obtenga un "corpus" suficientemente rico de enunciaciones en el nivel 3, y proceda a establecer el conjunto $M_{L}$.

\section{PASO 3:}

Obtenga un "corpus" suficientemente rico de enunciaciones en el nivel 4 y proceda a establecer el conjunto $\Sigma_{L}$ :

Cada "corpus" se obtiene y analiza sobre la base de las enunciaciones proporcionadas por uno o más "informantes nativos", y mediante la utilización de "procedimientos de campo" diseñados adecuadamente.

Sin entrar de lleno a señalar deficiencias intrínsecas en las definiciones anteriores (e. g. el hecho de que dos fonos en distribución complementaria no necesariamente pertenecen al mismo fonema- (3) ), hacemos notar lo siguiente:

1. Aunque se pretende proceder en forma estrictamente ascendente, el formalismo utilizado no puede ocultar el hecho de que constantemente se apela a conceptos o definiciones intuitivas de niveles superiores. En par- ticular, es notoria la presencia de implícitas suposiciones semánticas.

2. Aunque sin declararlo expresamente, los conjuntos utilizados en todas las definiciones y construcciones se suponen numerables. De hecho, toda la concepción subyacente parece estar estructurada sobre una "visión numerable" del lenguaje.

3. Aunque es evidente que la noción de oración aislada de un contexto es insostenible en el contexto real de los lenguajes naturales, esta noción se toma tácitamente como válida. No se consideran unidades lingüísticas de orden superior, a pesar de que son éstas las que constituyen la clase lingüística más importante en el tráfico lingü ístico ordinario. Por regla general, la gente no piensa ni se comunica en oraciones, sino en unidades discursivas más complejas, cuya coherencia estructural involucra necesariamente componentes semánticos.

Lo anterior no es en realidad una crítica especialmente novedosa: se ha hecho antes de una u otra manera al apuntar las deficiencias epistemológicas de la lingüística estructuralista. Más curioso es el hecho de que estas mismas observaciones pueden hacerse, en menor o mayor grado, a quienes utilizan la perspectiva de la gramática de transformaciones. Sin explorar detalladamente esta afirmación, es posible ver las implicaciones de las suposiciones tácitas que hemos señalado en la base de gran parte del pensamiento lingü ístico actual: basta con analizar algunas consecuencias operacionales.

En primer lugar, una "visión numerable" del lenguaje nos permite sustentar la validez de una muestra numerable de datos. En efecto, si el conjunto sobre el cual operamos es numerable, una muestra numerable puede ser representativa. Por el contrario, si este conjunto no fuera numerable, una muestra numerable carecería de representatividad. La suposición que hagamos sobre la "numerabilidad" del lenguaje tendrá consecuencias determinantes, puesto que hasta la fecha solo podemos trabajar con muestras seleccionadas.

Por otra parte, el suponer que podemos describir ciertos estratos del lenguaje sin necesidad de considerar la función significativa, no solo refuerza solapadamente la "visión numerable", sino que nos ahorra el molestísimo trabajo de tener que formalizar esta función. Sobre la inevitabilidad de hacer algún tipo de consideración semántica cuan- 
do se estudia cualquier estrato del lenguaje se ha escrito bastante. Quiero abocarme ahora al problema de la "numerabilidad lingü ística".

Curiosamente, este problema está ligado al de definir formalmente algún tipo de estructura de nivel superior al oracional. Comenzaré por intentar hacer precisamente eso.

\section{Definición:}

Un "pronunciamiento lingüístico" es un conjunto de asignaciones, donde una asignación se expresa típicamente mediante una oración del tipo "A $x$ le asigno $\mathrm{Y}$ " o, equivalentemente, "A $x$ le haré corresponder $y$ ".

\section{Ejemplo:}

Un calendario de trabajo es un pronunciamiento lingü ístico finito: $C A L-T R A=\{$ "De seis a siete me levantaré", "Desayunaré de siete a siete y media", "De siete y media a ocho viajaré al trabajo", "De ocho a once terminaré el informe sobre requisitos fiduciarios", "De once a doce me tengo que presentar a la reunión semanal", "De doce a una almorzaré", "De una a cuatro comenzaré el reporte de fideicomisos", "De cuatro a cuatro y media regresaré a la casa", "De cuatro y media a seis leeré el periódico", "Cenaré de seis a siete", "De siete a diez veré televisión", "De diez a seis dormiré". $\}$

\section{Teorema:}

El conjunto de todos los pronunciamientos lingü ísticos no es numerable.

\section{Demostración:}

Considérense todos los pronunciamientos lingù ísticos de la forma $\{\mathrm{Al}$ uno le asigno el uno, Al dos le asigno el uno, Al tres le asigno el cero,...., Al cuarenta millones doscientos veinte mil le asigno el cero,........... $\}$. En general, todos los pronunciamientos lingü ísticos cuyos elementos son de la forma "Al n le asigno x", con $n \in N$ y $x \in\{0,1\}$. Supongamos que el conjunto de todos estos pro- nunciamientos lingü ísticos es numerable y designémoslo mediante el símbolo $P$. Podemos entonces escribir $P=\left\{P_{1}, P_{2}, \ldots, P_{n}, \ldots ..\right\}$, donde $P_{j}$ es como se ha descrito antes, $j \in N$. Considérese ahora el pronunciamiento lingüístico $\mathrm{P}$ definido de la siguiente manera:

$$
\begin{aligned}
& \text { Para cada } \\
& \text { entero n, }
\end{aligned}\left\{\begin{array}{l}
- \text { "A } \mathrm{n} \text { le asigno el uno" si en } \mathrm{P}_{\mathrm{n}} \\
\text { tengo "A n le asigno el cero". } \\
\text { - "A n le asigno el cero" en cual- } \\
\text { quier otro caso. }
\end{array}\right.
$$

Entonces $P \notin Q$, puesto que se diferencia de cada $P_{j}$ en $P$. Esto es una contradicción, pues hemos supuesto que $Q$ contiene todos los pronunciamientos cuyos elementos son de la forma "A $m$ le asigno $x^{\prime \prime}, m \in N, x \in\{0,1\}$. Luego, la suposición de que $\mathcal{P}$ es numerable es una suposición falsa.

Hemos demostrado, pues, que el lenguaje tiene características de innumerabilidad. Las consecuencias de este resultado son considerables, como debería mostrar un breve re-examen de las observaciones hechas al respecto con anterioridad.

Ahora bien, podría argüirse que ningún pronunciamiento lingüístico en la realidad es de cardinalidad infinita. Esta, que no parece ser una objeción teórica de peso, puede responderse también directamente. En efecto, si nosotros procesáramos la información secuencialmente (es decir, en serie), tal posibilidad no podría darse en la práctica. Sin embargo, es un hecho que nosotros, a diferencia de los ordenadores electrónicos, procesamos la información simultáneamente (es decir, en paralelo). Es perfectamente razonable suponer que en muchas ocasiones nosotros hacemos una decisión (no necesariamente binaria) sobre un conjunto infinito de variables (posiblemente numerable). $\mathrm{Si}$, como afirman ciertos psicolingüistas modernos (4), existe una estructura esquemática mental que subyace tanto la integración de los preceptos como la integración de las producciones lingùísticas, no parece descabellado suponer la existencia de pronunciamientos lingü ísticos infinitos. 


\section{NOTAS}

(1) Fodor, Bever, Garrett. The Psychology of Language, McGraw-Hill, 1974, pág. 30.

(2) IDEM, pág. 31.

(3) El ejemplo dado por los autores antes citados es tomado del inglés, donde [ $\mathrm{h}$ ] y [ $\mathrm{J}$ ] aparecen distribuidos complementariamente sin ser, como es obvio, alófonos de un mismo fonema. Para resolver este tipo de problema, se le exige a dos fonos en distribución complementaria cumplir con otra condición para ser considerados alófonos: la de ser "acústicamente similares". De los problemas surgi- dos a partir de esta nueva restricción se habla ampliamente en las páginas 36-40.

(4) El libro entero de Fodor, Bever y Garrett se dedica a proporcionar un resumen de lo hecho hasta la fecha de 1974 en torno a una pregunta esencial del "mentalismo experimental": ¿qué procesos mentales están involucrados en la codificación y descodificación de la estructura sintáctica de las oraciones? El objeto de la psicolingüística "mentalista" es caracterizar los procesos mentales involucrados en la integración perceptual y conductual, para lo cual debe suponer la existencia de un contenido o esquema mental subyacente. 